\title{
Research Paper \\ The Effect of an 8-Week Selected Theraband Training on Balance and Motor Performance in Young Wrestlers
}

\author{
${ }^{*}$ Milad Sadeghi $^{1} \odot$, Reza Mahdavi Nejad ${ }^{2}$
}

1. Department of Corrective Exercise and Sport Injury, Faculty of Physical Education and Sport Sciences, Khorasgan Branch, Islamic Azad University, Esfehan, Iran 2. Department of Sport Injuries \& Corrective Exercise, Faculty of Physical Education and Sport Sciences, Esfehan University, Esfehan, Iran.

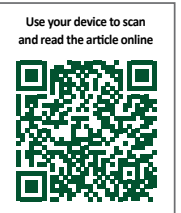

Chtation: Sadeghi M, Mahdavi Nejad R. [The Effect of an 8-Week Selected Theraband Training on Balance and Motor Performance in Young Wrestlers (Persian)]. Journal of Sport Biomechanics. 2019; 5(1):28-37. https://doi.org/10.32598/biomechanics.5.1.3

https://doi.org/10.32598/biomechanics.5.1.3

(a) 03

Article Info:

Received: 10 Nov 2018

Accepted: 27 Feb 2019

Available Online: 01 Jun 2019

Key words:

wrestling, Thera-

band, functional

movement screen,

Static Balance, Dy-

namic Balance

\section{ABSTRACT}

Objective Due to the recurrent and relatively high frequency of injuries in wrestling, identifying preventing strategies for such damages is of significant importance. The present study investigated the effect of 8 weeks of selected Thera-band exercises on the balance and functional movement of adolescent wrestlers.

Methods The study subjects included 30 athlete male wrestlers form Baft City, Iran, who were selected randomly and divided into two groups of exercise (age: $17.27 \mathrm{y}$, height: $1.72 \mathrm{~m}$, weight: $71.53 \mathrm{~kg}$, \& body mass index: $24.01 \mathrm{~kg} / \mathrm{m}^{2}$ ) and control (age: $17.07 \mathrm{y}$, height: $1.74 \mathrm{~m}$, weight: $72.46 \mathrm{~kg}$, and body mass index: 23.88 $\mathrm{kg} / \mathrm{m} 2$ ). A questionnaire was used to collect the study samples' demographic information and the history of sports. Moreover, to measure static and dynamic balance and functional movement, the stork test, Y balance test, and functional movement screen test were used, respectively. Independent Samples t-test, Paired Samples t-test, Mann-Whitney U-test, and Wilcoxon test were used to compare the mean score differences at $\mathrm{P}<0.05$.

Results The present study results suggested a significant difference in the static balance $(P<0.001)$ and the total dynamic balance $(P<0.001)$ between the two study groups.

Conclusion The collected data revealed that running a low-cost program could effectively improve balance and performance in the study samples. Furthermore, sports athletes, professional wrestlers, and freestyle wrestlers could use Thera-band training to enhance the motor pattern; such a measure could help to reduce the severity of damage and improve their movement patterns.

\section{Extended Abstract}

\section{Introduction}

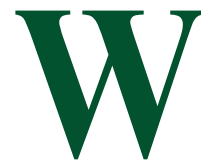

restling is among the most popular sports in Iran. The aggressive nature of wrestling results in $>9$ injuries per 1000 athletes [1]. In a study conducted at the Beijing Olympics, the overall prevalence of injuries was estimated to be 9.30 per 100 athletes and 7.88 per 100 competitions; of which, $84.4 \%$ of all cases were reported as mild injuries [2].

Due to the high incidence of injury in this sport, it is essential for these athletes to perform proper and regular training. Accordingly, all of their muscles will be involved to enhance their strength and endurance. Elastic band exercises

\section{* Corresponding Author:}

Milad Sadeghi, MSc.

Address: Department of Corrective Exercise and Sport Injury, Faculty of Physical Education and Sport Sciences, Khorasgan Branch, Islamic Azad University, Esfehan, Iran.

Tel: +98 (938) 7090067

E-mail: milad13697@gmail.com 
are considered as a safe tool. They are effective strategies for improving the neuromuscular system, muscle strength, and the ability to perform functional and balance tasks [8].

Han et al. performed a 4-week Theraband training among individuals with and without ankle sprains. Their collected results revealed that 4 weeks of balance training yielded significant improvements in both study groups [9]. Accordingly, this study aimed to investigate the effect of 8 weeks of Theraband selected exercises on balance and motor performance of young wrestlers in Baft City, Iran.

\section{Participants and Methods}

The statistical population of the present study consisted of all juvenile wrestlers in Baft City of Kerman Province, Iran. Like similar research literature, the athletes had $\geq 3$ years of experience and exercised $\geq 3$ sessions per week. Among them, 30 athletes (15 in the control group and 15 in the training group) were selected. To perform the dynamic balance test to explore the actual leg length, we measured the anterior-upper lumbar spine down to the medial ankle. This helped us to normalize the obtained data and gain a better comparison of the study subjects. We used the Y Balance Test to evaluate dynamic balance and Stork Balance Stand
Test to assess static balance [11]. The Functional Movement Screen (FMS) was also used to evaluate the basic movement pattern in the study subjects.

These inventories included 7 tests of deep squat, obstacle step, lunge, shoulder mobility, active leg lifting, trunk stability swimming, and rotational stability. The scoring method is that if a person made the correct move without compensatory movement, he/she would gain 3 points and if failed to make the compensatory movements or were unable to move, would obtain 2 and 1 points, respectively. Besides, if one felt pain during the movement, he/she would gain no points for that movement [7]. The training protocol of this study consisted of 8 weeks of Theraband exercises $[13,10]$.

\section{Results}

Comparing the pre-test scores of the two study groups indicated a significant difference between static balance $(\mathrm{t}=-1.10, \mathrm{P}=0.27)$, anterior directions $(\mathrm{t}=0.35, \mathrm{P}=0.72)$, internal posterior $(\mathrm{t}=-0.84, \mathrm{P}=0.40)$, external posterior $(\mathrm{t}=-$ $0.80, \mathrm{P}=0.42)$, and total dynamic balance score $(\mathrm{t}=-0.57$, $\mathrm{P}=0.56)$. Furthermore, there was no significant difference in motor function screening test values $(\mathrm{P}=0.21, \mathrm{Z}=-1.25)$

Table 1. Independent Samples t-test results for comparing the two groups at post-test phase

\begin{tabular}{|c|c|c|c|c|c|c|c|}
\hline Variables & Group & df & Mean Difference & $t$ & $\mathbf{P}$ & Lower & Upper \\
\hline Stork test & $\begin{array}{l}\text { Control } \\
\text { Experimental }\end{array}$ & 28 & -6.21 & -5.11 & $0.001^{*}$ & -8.69 & -3.72 \\
\hline $\begin{array}{l}\text { Anterior direction of the Y Balance Test (\% } \\
\text { of lower limb length) }\end{array}$ & $\begin{array}{c}\text { Control } \\
\text { Experimental }\end{array}$ & 28 & -5.92 & -2.35 & 0.02 & -11.07 & -0.76 \\
\hline $\begin{array}{l}\text { The internal posterior direction of the } Y \\
\text { Balance Test (\% of lower limb length) }\end{array}$ & $\begin{array}{l}\text { Control } \\
\text { Experimental }\end{array}$ & 28 & -7.37 & -4.80 & $0.001^{*}$ & -10.52 & -4.23 \\
\hline $\begin{array}{l}\text { External posterior direction of the Y Bal- } \\
\text { ance Test (\% of lower limb length) }\end{array}$ & $\begin{array}{l}\text { Control } \\
\text { Experimental }\end{array}$ & 28 & -4.46 & -2.14 & 0.04 & -8.74 & -0.19 \\
\hline $\begin{array}{l}\text { The Y Balance Test overall score } \\
\text { (\% of length) }\end{array}$ & $\begin{array}{l}\text { Control } \\
\text { Experimental }\end{array}$ & 28 & -6.16 & -4.71 & $0.001^{*}$ & -8.84 & -3.48 \\
\hline
\end{tabular}

\begin{tabular}{|c|c|c|c|c|c|}
\hline Variable & Group & No. & U & $\mathbf{z}$ & $\mathbf{P}$ \\
\hline \multirow{2}{*}{$\begin{array}{l}\text { Total score of Motor } \\
\text { Performance Test (FMS) }\end{array}$} & Control & 15 & \multirow{2}{*}{27} & \multirow{2}{*}{3.60} & \multirow{2}{*}{0.001} \\
\hline & Experimental & 15 & & & \\
\hline
\end{tabular}

* Significant at the 0.01 level. 
in the control and experimental groups. As per Table 1, the Theraband exercise protocol significantly impacted balance and motor performance in the studied samples.

According to Table 1, in the post-test, there was a significant difference between the control and experimental groups. This finding reflects the effect of exercise on balance and motor performance.

\section{Discussion}

that the obtained data revealed the selected exercises of Theraband were associated with improved static and dynamic balance in the investigated wrestlers; thus, the collected results were consistent with those of research studies in different sports fields and different groups with and without injury. Khodabakhshi et al. reported the positive impact of these exercises on the dynamic balance of young footballers [14]. Han et al. also emphasized the positive effect of 4 weeks of Theraband resistance training on improving dynamic balance in both groups of with and without chronic ankle instability [9]. The current study results were in line with those of other research studies regarding the effect of Theraband resistance training on balance.

The increased balan cially essential in the lower limbs, deep receptor activity, and neuromuscular control. It also leads to maintaining balance in the course of action and gaining the most scores. Therefore, one of the reasons for the improvement of balance as a result of resistance training in this study can be related to the increase in strength of lower extremities of the study subjects after participating in resistance training protocol.

\section{Conclusion}

The current study results suggested that the selected Theraband resistance training program could improve the static and dynamic balance in the studied wrestlers. The intervention also increased the scores of those who are susceptible to impaired functional movement screening. Therefore, it seems that athletes in this field, along with specialized wrestling and free weights-lifting training, could also use Theraband training to improve the movement pattern and activate muscles throughout the range of motion. This helps to reduce injury and improve their movement patterns.

\section{Ethical Considerations}

\section{Compliance with ethical guidelines}

Informed consent was obtained from the all participants and they were informed of the study method and objectives. They also were assured of the confidentiality of their personal information and were free to leave the study at any time

\section{Funding}

This study was extracted from the $\mathrm{PhD}$. thesis of the first author approved by the Department of Corrective Exercise and Sport Injury at Islamic Azad University of Khorasgan branch, and received no financial support from any organization.

\section{Authors' contributions}

Conceptualization, methodology, original draft preparation, editing \& review: Milad Sadeghi and Reza Mahdavi Nejad; Resources: Milad Sadeghi; Visualization, supervision, project administration: Reza Mahdavi Nejad.

\section{Conflicts of interest}

The authors declare no conflict of interest. 


\title{
تأثير هشت هفته تمرينات منتخب تراباندبر تعادل وعملكرد حركتى كثتى
}

1. كروه آسيبشناسى ورزشى و حركات اصلاحى، دانشكده تربيتبندى و علوم ورزشى، واحد خوراسكان، دانشعاه آزاد اسلامى، اصفهان، ايران.

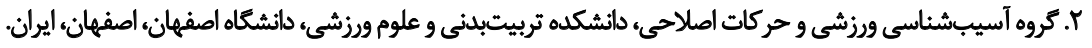

\begin{abstract}
حكيد

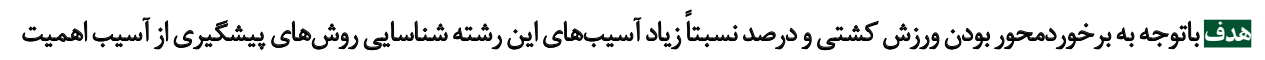

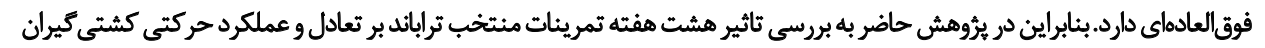

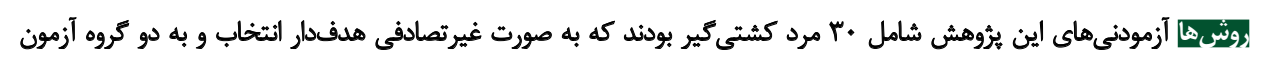

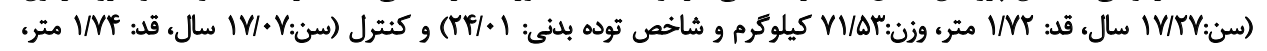

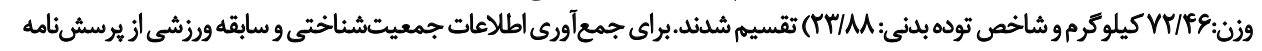

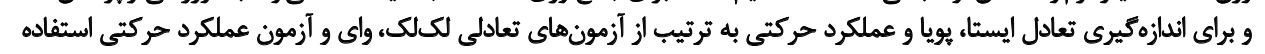

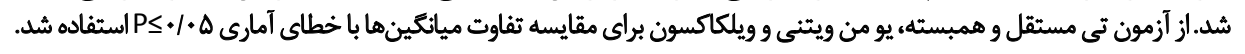

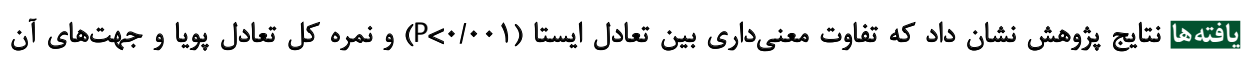

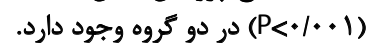

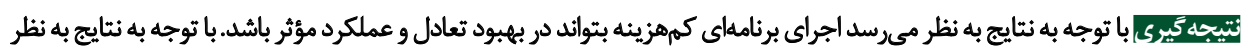

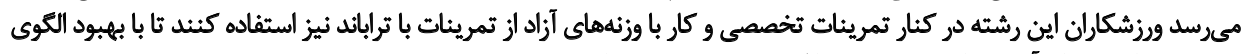

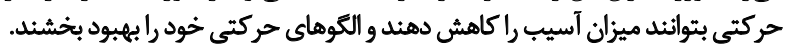

|طلاعات مقاله:

تاريخ دريافت: 19 آبان

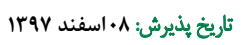

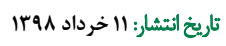

كليدوازٔهها:

كشتى، تراباند، آزمون عملكرد حركتي، تعادل

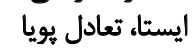

ثروهشهاى كشورهاى اسكانديناوى نشاندهنده اين است

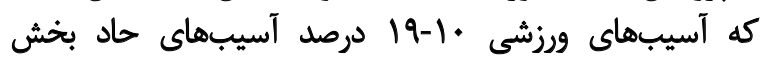

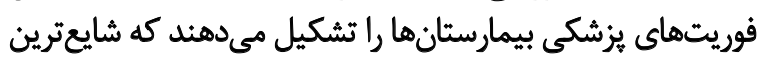

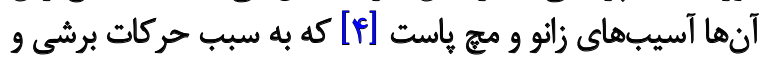

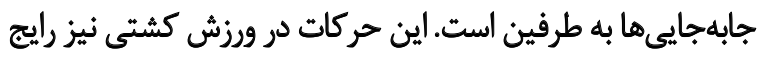

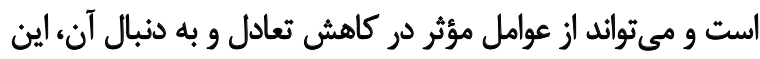

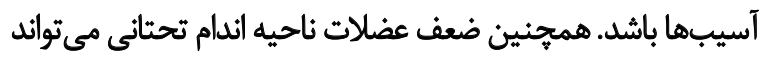

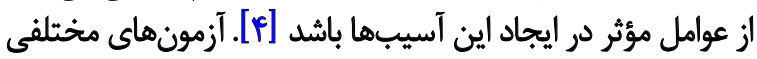

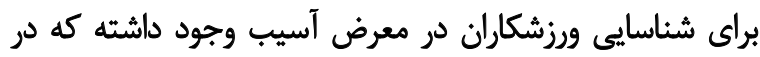

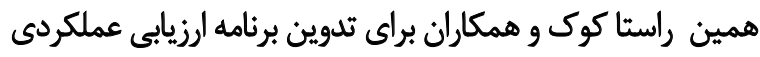

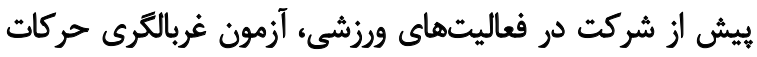

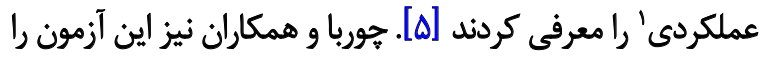

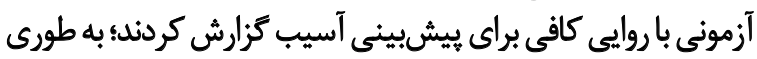

بدون شك كشتى از يرطرفدارترين و مردمىثرين رشتهائ ئراي

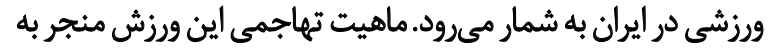

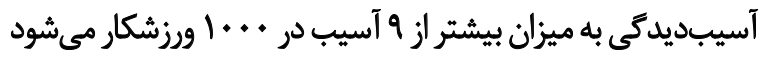

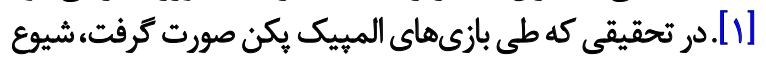

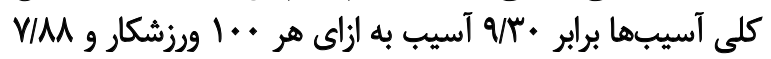

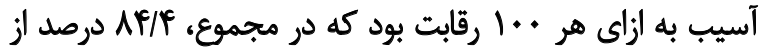

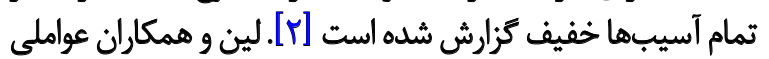

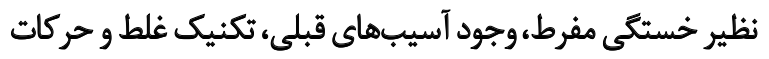

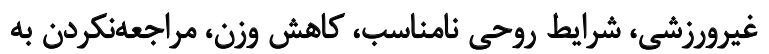

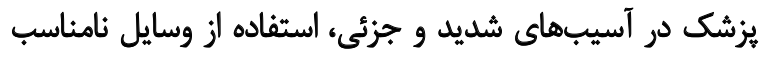

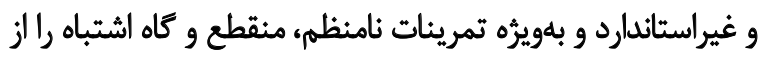

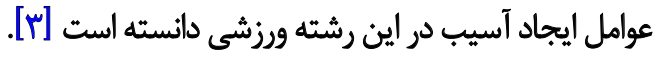

1. Functional movement Screening Test (FMS)

\section{-}

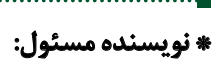

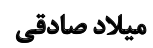

$$
\begin{aligned}
& \text { تشائى: اصفهان، دأشكاه آزاد اسلامي، واحد خورواسكان، دانشكده تربيتبدنى و علوم ورزشى، كروه آسيبشناسى ورزشى و حركات اصلاحى. }
\end{aligned}
$$

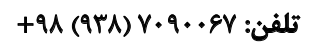

$$
\begin{aligned}
& \text { milad13697@gmail.com يست الكترونيكي: توبهن }
\end{aligned}
$$


كدام اندام تحتانى تمايل بيشترى براى زدن شوت فوتسال دارد، ياي

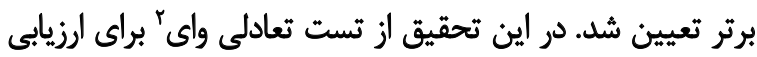

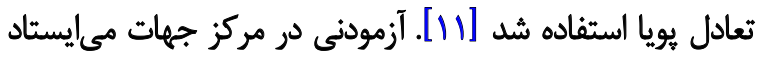

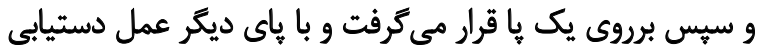

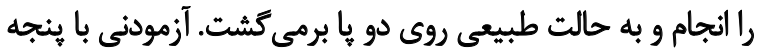

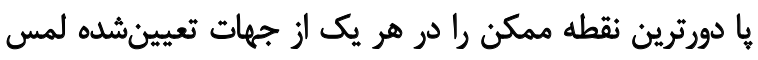

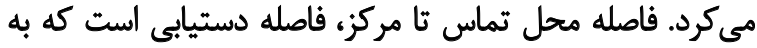

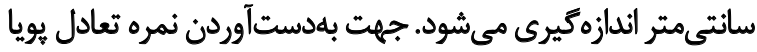

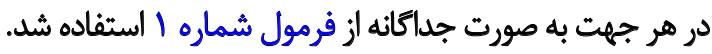

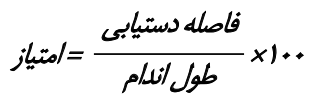

براى ارزيابى تعادل ايستا از آزمون لكلى استفاده شد. نحوه انجام

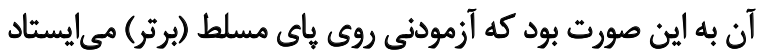

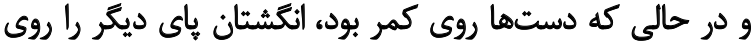

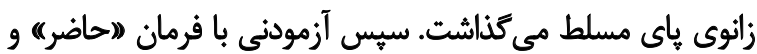

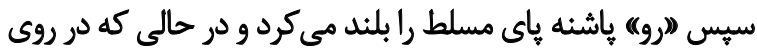

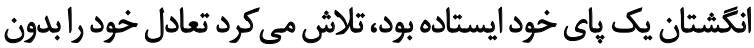

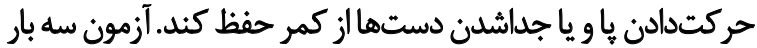

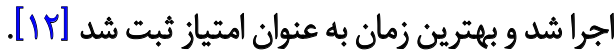
همجينين جهت ارزيابى الكوى حركات بنيادى از تست غربالكَرى

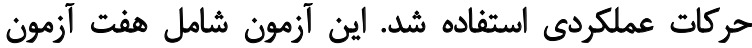

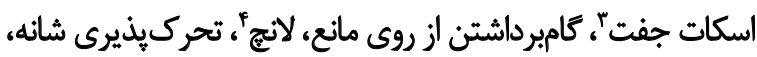

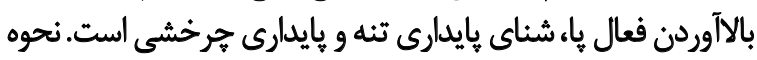

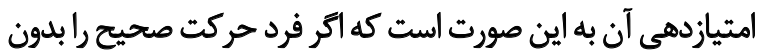

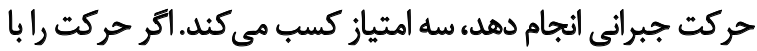

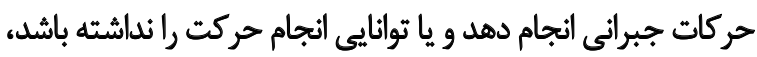

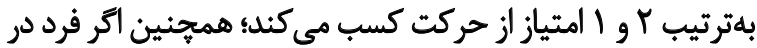

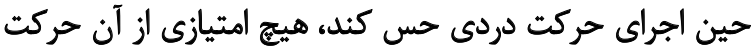

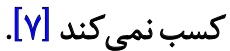

بروتكل تمرينى تحقيق حاضر شامل هشت هفته تمرينات تراباند

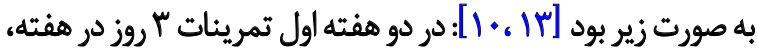

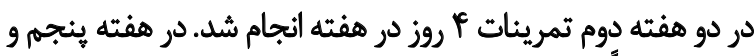

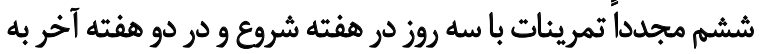
ههار روز در هفته افزايش يافت. مقاومت تراباندها به ترتيب هفته: صورتى: هفته اول (•D درصد)، هفته دوم (V) درصد) )هفته سوم و جهارم (• • ( درصد) همئه

\section{Y}

3. Deep squat

4. Lunge

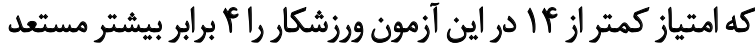

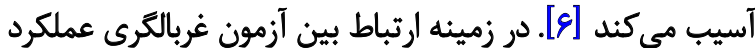

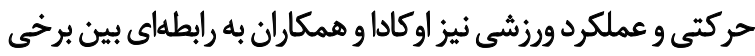

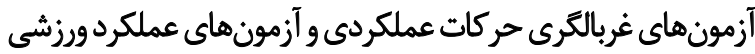

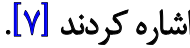

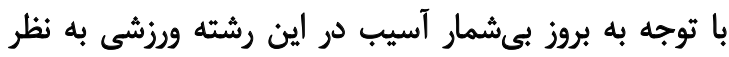

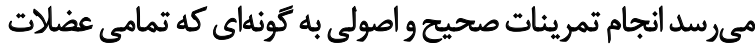

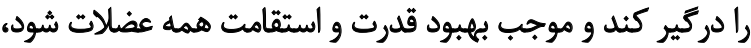

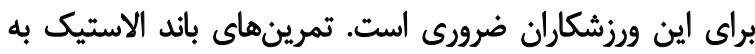

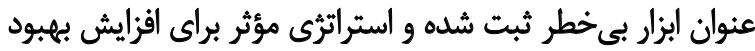

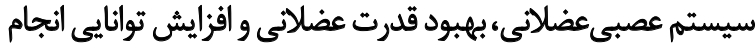

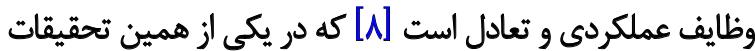

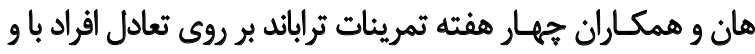

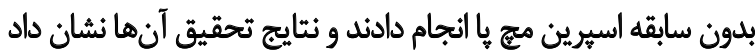

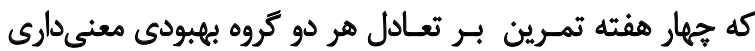

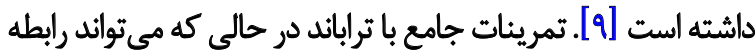

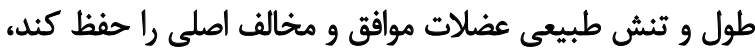

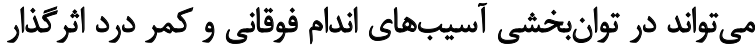

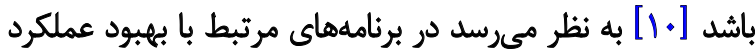

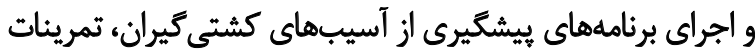

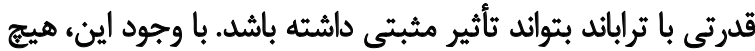

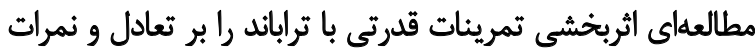

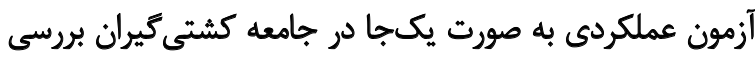

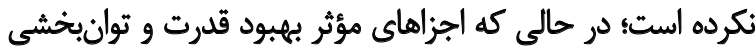

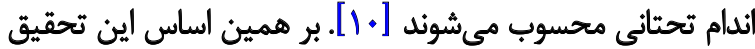

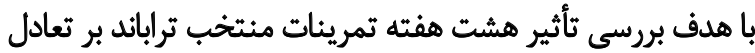

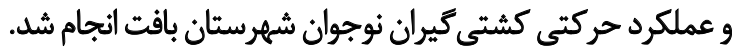

$$
\text { و }
$$

جامعه آمارى تحقيق شامل كليه كشتى كيران نوجوان شهر بافت

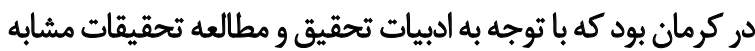

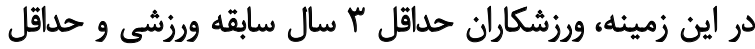

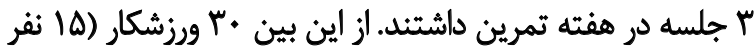

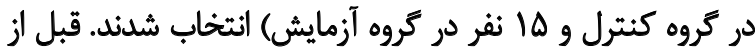

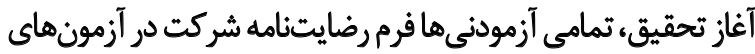

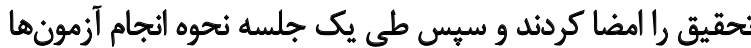

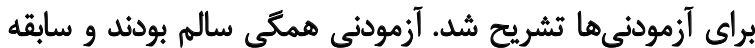

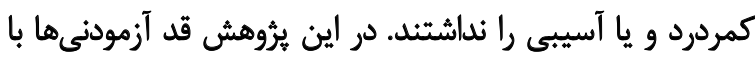

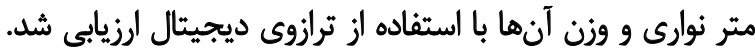

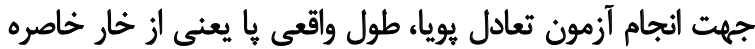

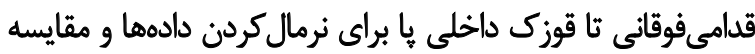

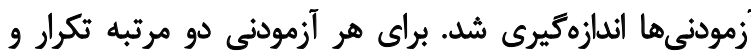

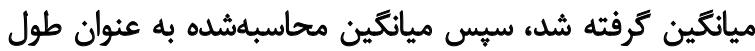

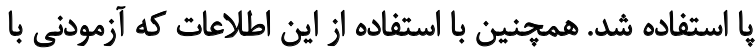




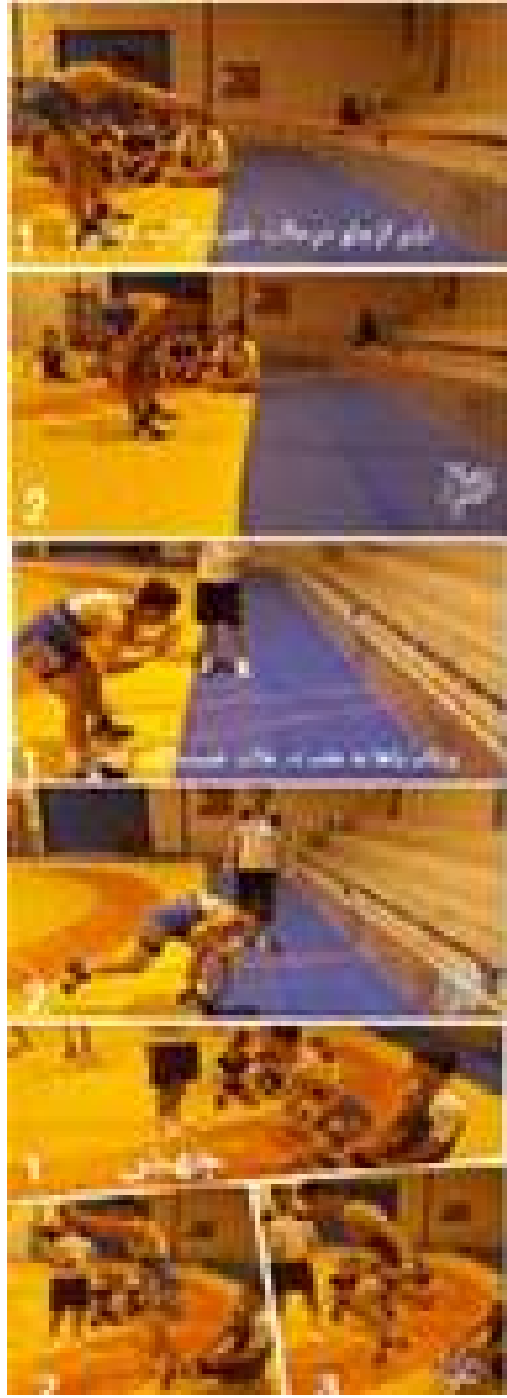

مجله بيومكانيك وزنش

كليه عمليات آمارى به وسيله نرمافزار SPSS نسخه זب انجام شد.

ثنانج

ميانكين و انحراف استاندارد مشخصات فردى آزمودنىها شامل

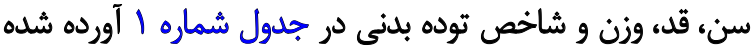

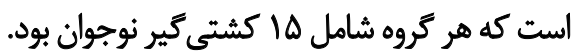

با توجه به نرمال بودن دادههاى تعادل ايستا و يويا كه با آزمون

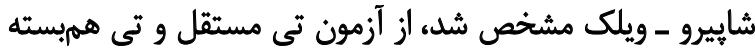

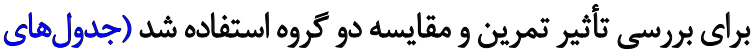

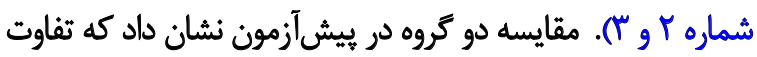

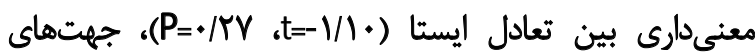

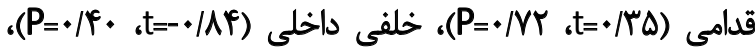

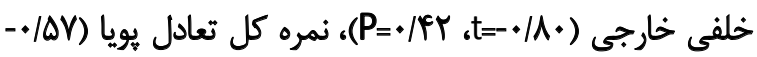

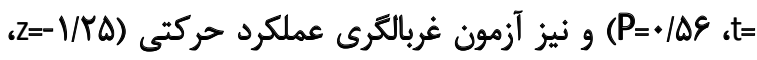

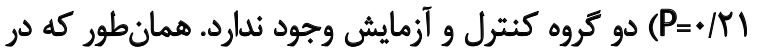

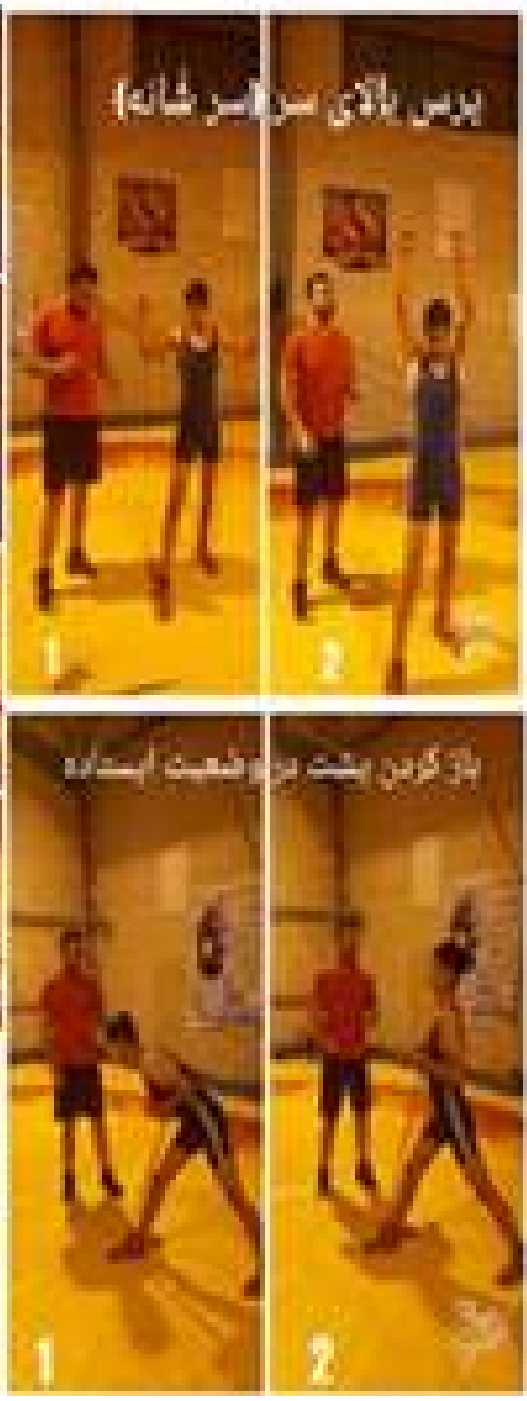

تصوير ا. بروتكل تمرينى تراباند نارنجى: هفته يثنجم (•D درصد)، هفته ششم (ه V درصد)، هفته

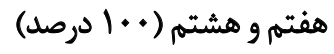

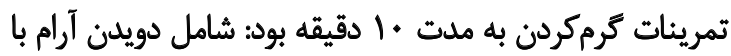

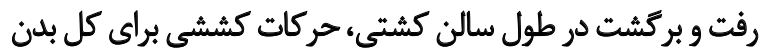

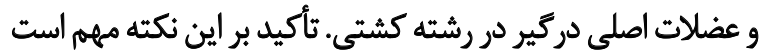

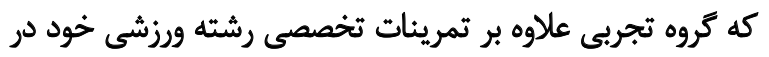

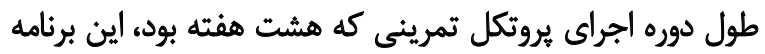
تمرينى را انجام مي دادئد (تصوير شماره ()). تهت تجزيه و تحليل اطلاعات جمعآورىشده ازئ روشهاى آمار آمار

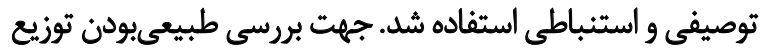

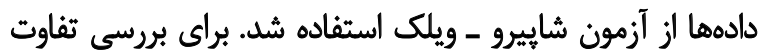

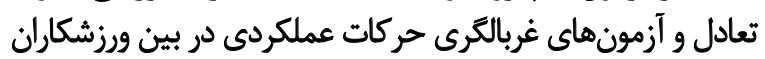

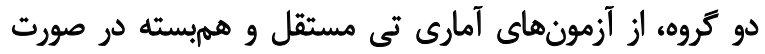

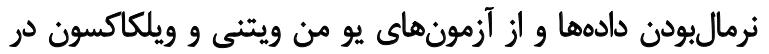

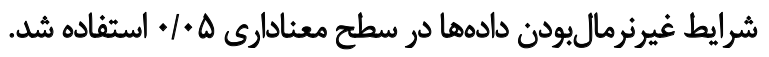


جدول ا. شاخص هاى آنترويومتريك مربوط به دو كروه

\begin{tabular}{|c|c|c|c|c|}
\hline $\mathbf{P}$ & $\mathbf{T}$ & ميانكيند|نحراف استاندارد & كروب & شاخص اندازه كيرى \\
\hline$+/ 49$ & $-+/ 8 \lambda$ & 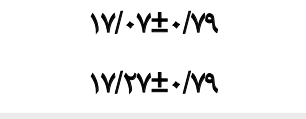 & كثترل آزمايش & سن (سال) \\
\hline . & $1 / \cdot 1$ & 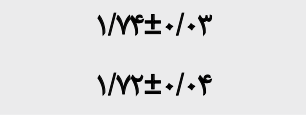 & كتّل آزمايش & قد (متر) \\
\hline ./Va &.$/ \pi T$ & $\begin{array}{l}V / \mathscr{R} \pm V / \& \Lambda \\
V / \Delta r \pm N R V\end{array}$ & كنترل & 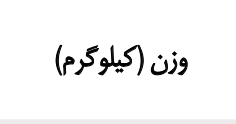 \\
\hline$+/ 9$. & $-+/ 4$ & $\begin{array}{l}r r / M \pm r / \Delta r \\
r Y / \cdot I \pm r / 19\end{array}$ & كتترل & (كيلوكرم بر مترد مربع) \\
\hline +198 & $-+/ 4 F$ & $\begin{array}{l}q / * \pm 1 / 9 r \\
q / \pi+ \pm r / 19\end{array}$ & كثترل & سابقه ورزشى (سال) \\
\hline
\end{tabular}

مجله بيومكانيك وزنش

جدول شماره r نشان داده شده يروتكل تمرينى تراباند بر تعادل و بر عوامل تعادل و عملكرد حركتى است. عملكرد حركتى تأثير معنى دارى داشته است.

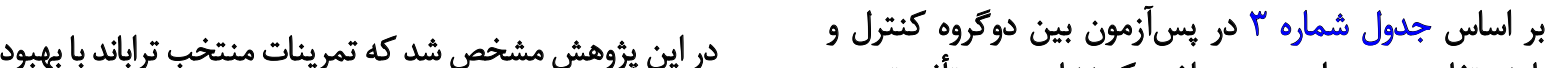

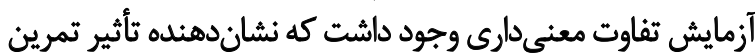

جدول r. تفاوت ميانكين عوامل در آزمودنىها قبل و بعد از اعمال يروتكل تمرينى (ه نفر)

\begin{tabular}{|c|c|c|c|c|c|c|c|c|}
\hline \multicolumn{8}{|c|}{ ميانكين \انحراف استاندارد } & \multirow{3}{*}{ تروه } \\
\hline \multicolumn{4}{|c|}{ 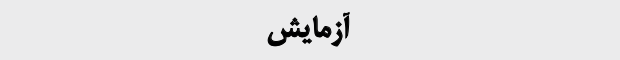 } & \multicolumn{4}{|c|}{ كتثرل } & \\
\hline $\mathbf{P}$ & $\mathbf{T}$ & يس آزمون & يريش آزمون & $\mathbf{P}$ & $\mathbf{T}$ & يسأزمون & يبيش آزمون & \\
\hline$+/ .+)^{* *}$ & - & $r F / T Y \pm r / \Delta \Delta$ & $19 / 1 r \pm r / / V$ &.$/ 94$ & $-+/ 4$ & $W \cdot F \pm r / \cdot V$ & $\mid V / a f \pm r / q g$ & تعادل ايستا \\
\hline $.1 . .1^{* *}$ & $-N+T$ & $A V / 8 . \pm N 1$ & $\Lambda \cdot 1 \cdot 9 \pm N \cdot V$ & .1 .8 & $-1 / 41$ & $\Lambda V / \& \Lambda \pm \Delta / F 1$ & $A \cdot / q \uparrow \pm \Delta / / \Delta$ & (درصد طول قدامى أزمون وائى \\
\hline $.1 ..)^{m *}$ & $-8 / P A$ & 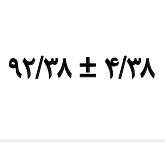 & $N E / N F \pm / M$ & .1 .8 & $-r / \cdot f$ & $\Lambda \Delta / . . \pm \pm F / \cdot r$ & 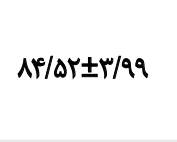 & 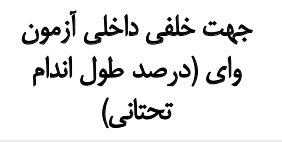 \\
\hline$+1+1^{* *}$ & $-r / x q$ & $\Delta r / \Lambda+ \pm r / \Lambda r$ & $r q / 11 \pm r /+r$ &.$/ 1 r$ & $-1 / 81$ & VNGT \pm S/9F & $W / \& 9 \pm \Delta / \& \Delta$ & 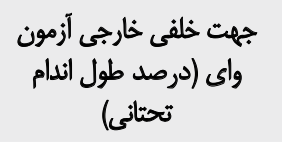 \\
\hline$+\%+1^{* *}$ & $-1 \pi / N$ & AV/qq $\pm r / \pi r$ & $\Lambda 1 / W \pm r / 4 \varepsilon$ & $+1+\Delta V$ & $-r /+Y$ & $\Lambda / \Delta r \pm r / q$. & $\Lambda \mathrm{N} /+F \pm r / F F$ & نمره كلى أزمون واى (درصد \\
\hline \multicolumn{4}{|c|}{ 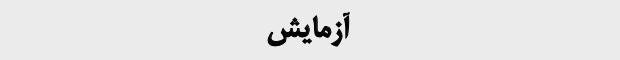 } & & & كتثرل & & \multirow{2}{*}{ 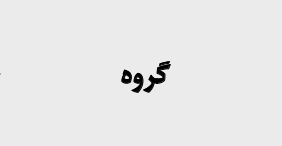 } \\
\hline $\mathbf{P}$ & $\mathbf{Z}$ & يس آزمون & بيشآزمون & $\mathbf{P}$ & $\mathbf{Z}$ & 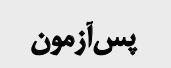 & بيش آزمون & \\
\hline $.1+1^{* *}$ & $-r / r \mid$ & $\mid \Delta / \& \& \pm V / F$ & $1 \Delta / r+ \pm 1 / r$ &.$/ \mathrm{V}$ & $-1 / r \Delta$ & $\mid r / q r \pm r / \cdot \Delta$ & $\mid r / T \cdot \pm Y / \cdot \varphi$ & 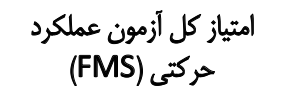 \\
\hline
\end{tabular}


جدول ץ. نتايج آزمون تى مستقل براي مقايسه دو كروه در يسآزمون

\begin{tabular}{|c|c|c|c|c|c|c|c|}
\hline Upper & Lower & $\mathbf{P}$ & $\mathbf{t}$ & اختلاف مياتغين & df & كروه & مثغير \\
\hline$-r / r r$ & $-N E q$ & $+1+.1^{* *}$ & $-\Delta / 11$ & $-8 / \pi 1$ & ru & كنترل & آزمون لكلى \\
\hline.$- / v E$ & $-11 / \cdot V$ &.$/+r^{*}$ & $-r / T \pi \Delta$ & $-\Delta / 9 r$ & ru & كثترل & 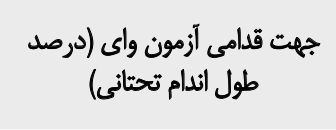 \\
\hline W/T & $-1+/ \Delta r$ & $+1+\infty)^{* *}$ & $-r / \lambda$ & $-V / \pi$ & ru & كنترل & 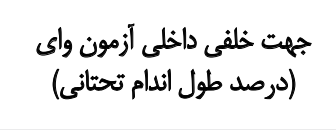 \\
\hline.$- / 19$ & $-N V^{e}$ & $.1 . e^{e *}$ & $-T / 1 F$ & $-r / f e$ & rA & كنترل & 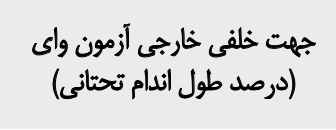 \\
\hline$-r / F A$ & -NAF & $.1 * .1^{* *}$ & $-r / M$ & $-8 / 18$ & rA & كنترل & نمره كلى آزمون واي (درصد طول \\
\hline
\end{tabular}

\begin{tabular}{|c|c|c|c|c|c|}
\hline $\mathbf{P}$ & $\mathbf{Z}$ & $\mathbf{U}$ & $\mathbf{N}$ & كروه & مثغير \\
\hline $.1 * .1^{* *}$ & $-r / \varepsilon$. & TV & 10 & كنترل & $\begin{array}{c}\text { امتياز كل آزمون عملكرد حركتي } \\
\text { (FMS) }\end{array}$ \\
\hline
\end{tabular}

مجله بيومكانيك وزنشي

"منى دارى در سطح هـ"

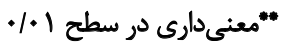

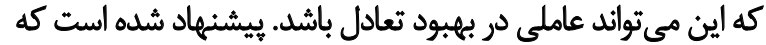

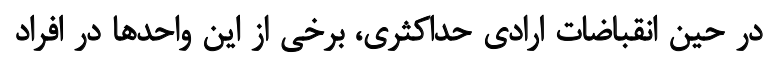

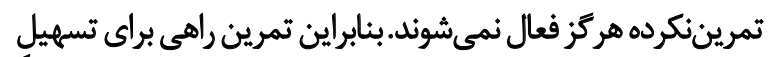

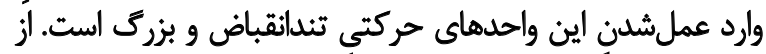

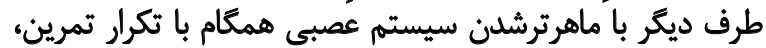

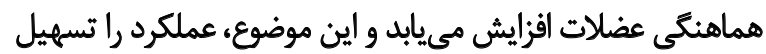

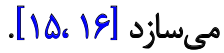

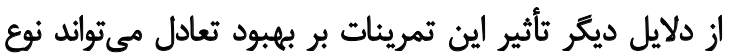

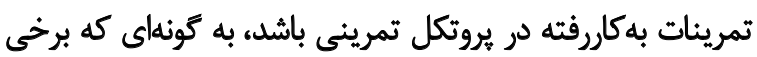

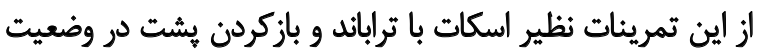

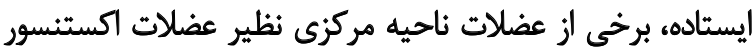

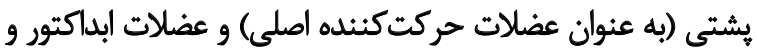

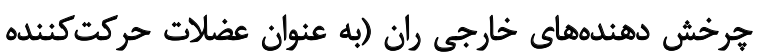

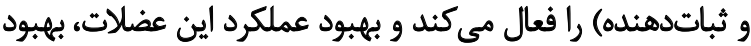

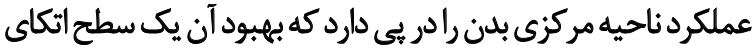

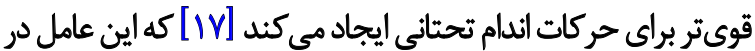

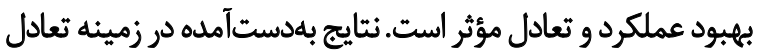

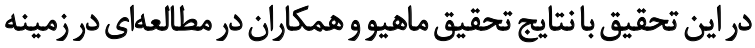

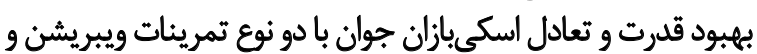

تعادل ايستاو يوياي كشتى كيران همراه بوده است.نتايج بهدستآمده

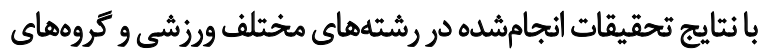

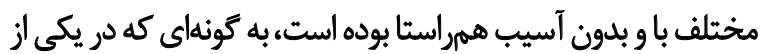

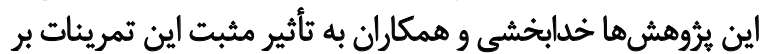

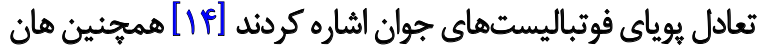

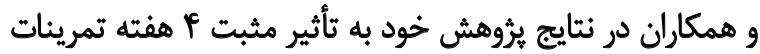

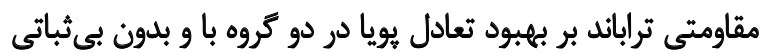

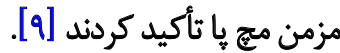
همسو بودن نتايج تحقيق حاضر با نتايج تحقيقات ديكر دليل

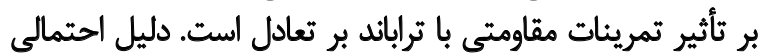

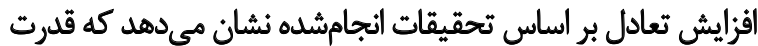

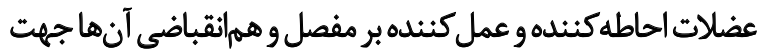

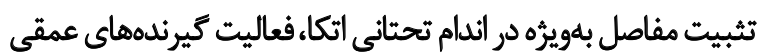

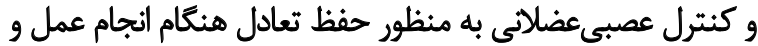

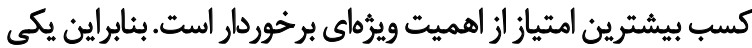

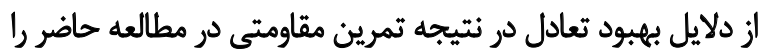

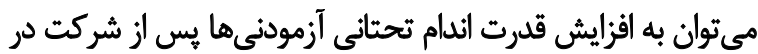

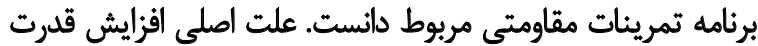

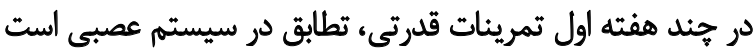




\section{نتبجدئيرى نهايي}

به صورت كلى نتايج اين مطالعه نشان داده كه برنامه تمرينى

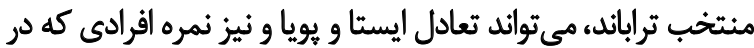

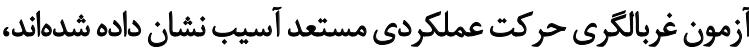

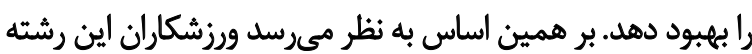

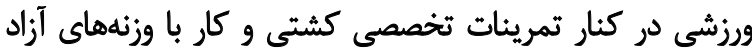

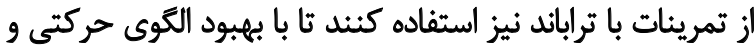

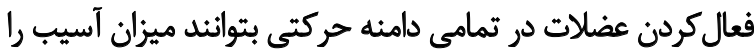

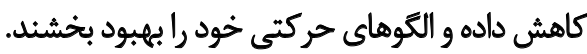

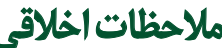

$$
\text { يبيروى از اصول اخلاق بثوهش }
$$

تمامى آزمودنىهاى تحقيق حاضر با رضايت كامل در برؤوهش

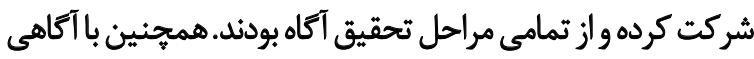

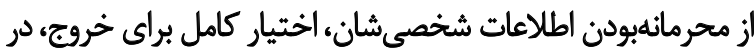
هريك از مراحل تحقيق راداشتيند.

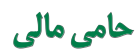

اين مقاله از قاياننامه آقاى ميلاد صادقى از گروه آسيبشناسى

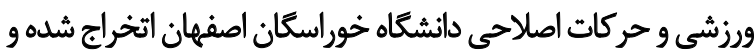
از حمايت مالى هيج بخشى استفاده نكرده است.

$$
\text { مشاركت نويسندكًان }
$$

مفهومسازى و تحقيق و بررسى: ميلاد صادقى و دكتر رضا

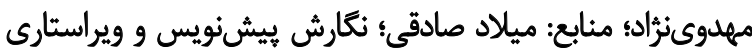

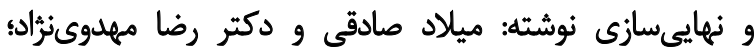

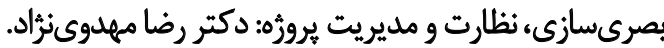

$$
\text { تعارض منافع }
$$

بنا به اظهار نويسندكان، اين مقاله هيج تعارض منافعى وجود ندارد.
مقاومتى همراستانبود، زيرا نتايج تحقيق آنهانشان داد كه هيجيجدام

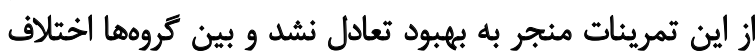

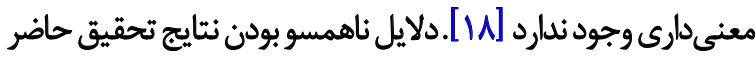

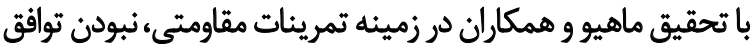

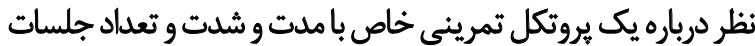

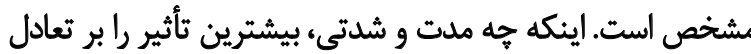

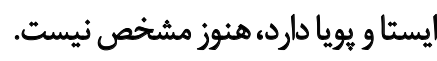
نتايج تحقيق حاضر در زمينه عملكرد حركتى نشاندهنده تأثير

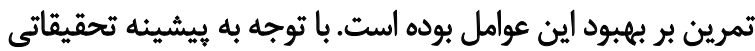

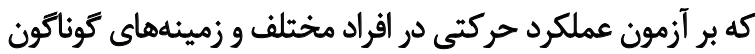

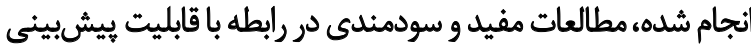

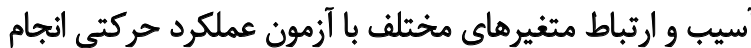

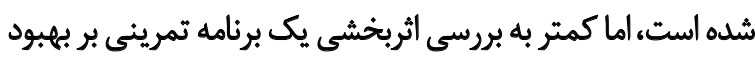

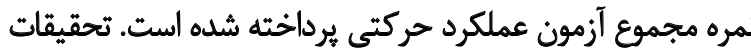

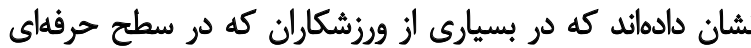

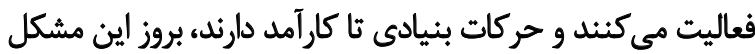

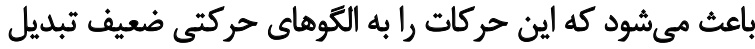

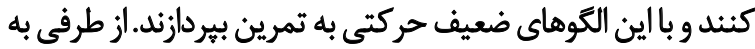

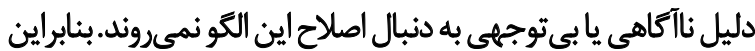

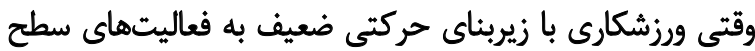

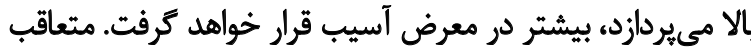

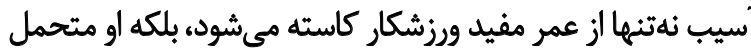

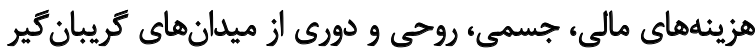

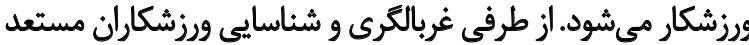

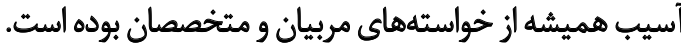

در اين بروهش نتايج بهدست آمده در زمينه آزمونهاى مربوط به عملكرد حركتى نشان داد كه تمرينات منتخب ترئ تراباند به به عنوان

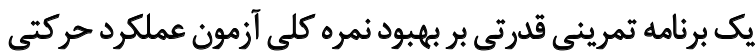

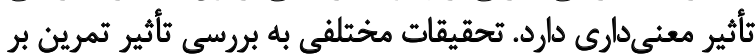

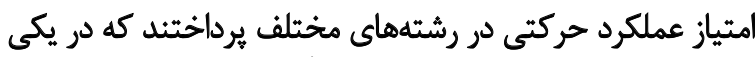

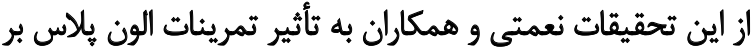

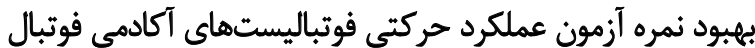

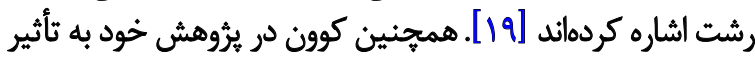

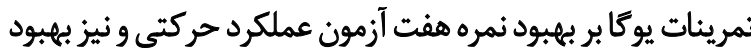

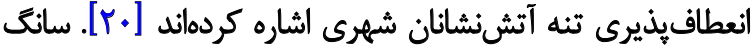

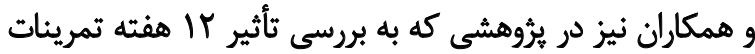

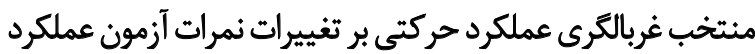

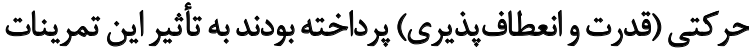

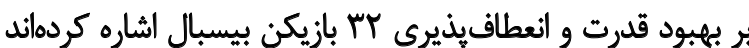

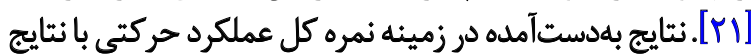

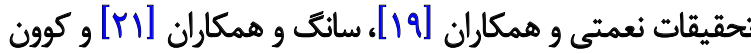

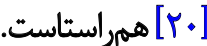




\section{References}

[1] Hewett TE, Pasque C, Heyl R, Wroble R. Wrestling injuries. Medicine and Sport Science. 2005; 48:152-78. [DOI:10.1159/000084288] [PMID]

[2] Junge A, Engebretsen L, Mountjoy ML, Alonso JM, Renström PA, Aubry MJ, et al. Sports injuries during the Summer Olympic Games 2008. The American Journal of Sports Medicine. 2009; 37(11):2165-72. [DOI:10.1177/0363546509339357] [PMID]

[3] Lin ZP, Chen YH, Chia F, Wu HJ, Lan LW, Lin JG. Episodes of injuries and frequent usage of traditional Chinese medicine for Taiwanese elite wrestling athletes. The American Journal of Chinese Medicine. 2011; 39(2):233-41. [DOI:10.1142/S0192415X11008774] [PMID]

[4] Bahr R, Krosshaug T. Understanding injury mechanisms: A key component of preventing injuries in sport. British Journal of Sports Medicine. 2005; 39(6):324-9. [DOI:10.1136/bjsm.2005.018341] [PMID] [PMCID]

[5] Cook G, Burton L, Hoogenboom B. Pre-participation screening: The use of fundamental movements as an assessment of function - part 1. North American Journal of Sports Physical Therapy. 2006; 1(2):62-72. [PMID] [PMCID]

[6] Chorba RS, Chorba DJ, Bouillon LE, Overmyer CA, Landis JA. Use of a functional movement screening tool to determine injury risk in female collegiate athletes. North American Journal of Sports Physical Therapy. 2010; 5(2):47-54. [PMID] [PMCID]

[7] Okada T, Huxel KC, Nesser TW. Relationship between core stability, functional movement, and performance. The Journal of Strength \& Conditioning Research. 2011; 25(1):252-61. [DOI:10.1519/ JSC.0b013e3181b22b3e] [PMID]

[8] Ciolac E, Garcez-Leme L, Greve J. Resistance exercise intensity progression in older men. International Journal of Sports Medicine. 2010; 31(6):433-8. [DOI:10.1055/s-0030-1249087] [PMID]

[9] Han K, Ricard MD, Fellingham GW. Effects of a 4-week exercise program on balance using elastic tubing as a perturbation force for individuals with a history of ankle sprains. Journal of Orthopaedic \& Sports Physical Therapy. 2009; 39(4):246-55. [DOI:10.2519/jospt.2009.2958] [PMID]

[10] Dashti P, Shabani M, Moazami M. [Comparison of the effects of two selected exercises of Theraband and Pilates on the balance and strength of lower limb in elderly women (Persian)]. The Iranian Journal of Obstetics, Gynecology and Infertility. 2015; 18(153):1-9.

[11] Jelinek HF, Khalaf K, Poilvet J, Khandoker AH, Heale L, Donnan LA. The effect of ankle support on lower limb kinematics during the $Y$-balance test using nonlinear dynamic measures. Frontiers in Physiology. 2019; 10:935. [DOI:10.3389/fphys.2019.00935] [PMID] [PMCID]

[12] Reiman MP, Manske RC. Functional testing in human performance. Champaign: Human Kinetics; 2009.

[13] Erfanian Zorufi F, Moazzami M, Mohamadi MR. [The effect of resistance training on static balance and pain in elderly women with varus knee and osteoarthritis by using elastic band (Persian)]. Journal of Paramedical Sciences \& Rehabilitation. 2016; 5(2):14-24. [DOI:10.22038/ JPSR.2016.6907]

[14] Khodabakhshi M, Hashemi Javaheri SAA, Ebrahimi Atri A, Ebadi Fara $M$. [Effects of 8 weeks of resistance training with traband on dynamic balance in young soccer players (Persian)]. Journal of Sport Biomechanics. 2016; 2(2):43-53.

[15] Wikstrom EA, Powers ME, Tillman MD. Dynamic stabilization time after isokinetic and functional fatigue. Journal of Athletic Training. 2004; 39(3):247-53. [PMID] [PMCID]
[16] Necking L, Lundborg G, Friden J. Hand muscle weakness in longterm vibration exposure. Journal of Hand Surgery. 2002; 27(6):520-5. [DOI:10.1054/jhsb.2002.0810] [PMID]

[17] Mok NW, Yeung EW, Cho JC, Hui SC, Liu KC, Pang CH. Core muscle activity during suspension exercises. Journal of Science and Medicine in Sport. 2015; 18(2):189-94. [DOI:10.1016/j.jsams.2014.01.002] [PMID]

[18] Mahieu NN, Witvrouw E, Van de Voorde D, Michilsens D, Arbyn V, Van den Broecke W. Improving strength and postural control in young skiers: Whole-body vibration versus equivalent resistance training. Journal of Athletic Training. 2006; 41(3):286-93. [PMID] [PMCID]

[19] Nemati N, Norasteh AA, Alizadeh MH. The effect of FIFA+ 11 program on functional movement screen scores of junior soccer players. Annals of Applied Sport Science. 2017; 5(3):23-9. [DOI:10.29252/acadpub.aassjournal.5.3.23]

[20] Cowen VS. Functional fitness improvements after a worksite-based yoga initiative. Journal of Bodywork and Movement Therapies. 2010; 14(1):50-4. [DOI:10.1016/j.jbmt.2009.02.006] [PMID]

[21] Song HS, Woo SS, So WY, Kim KJ, Lee J, Kim JY. Effects of 16-week functional movement screen training program on strength and flexibility of elite high school baseball players. Journal of Exercise Rehabilitation. 2014; 10(2):124-30. [DOI:10.12965/jer.140101] [PMID] [PMCID] 